\title{
Innovations in electromembrane processes
}

\author{
Krzysztof MITKO, Marian TUREK
}

Silesian University of Technology, Faculty of Chemistry, Gliwice, Poland

\begin{abstract}
Electromembrane processes are increasingly important group of separation methods, widely used for removal of charged components from solutions. It is a growing field of research with a plethora of both existing and still developed applications. The separation is based on ion migration across the charged membranes (a polymeric matrix with fixed charged groups, counterbalanced with mobile counter-ions), placed in the electric field. This paper presents the main electromembrane processes: electrodialysis (ED), electrodialysis reversal (EDR), electrodialysis with bipolar membrane (EDBM), electrodeionization (EDI), membrane capacitive deionization (MCDI), reverse electrodialysis (RED). We present the common applications of electromembrane processes and discuss the physical basis of the electromembrane processes. The most important parameters of the ion-exchange membranes are discussed, as well as the novel approaches towards mitigation of scaling, enhancement of mass transfer, decreasing the concentration polarization, and new hybrid electromembrane processes. Critical analysis of the possibility of energy production by reverse electrodialysis is presented.
\end{abstract}

Keywords: electrodialysis; electrodialysis reversal; reverse electrodialysis; electrodeionization; membrane capacitive deionization

Electromembrane processes are increasingly important group of separation methods, widely used for removal of charged components from solutions. The separation is based on ion migration across the charged membranes (a polymeric matrix with fixed charged groups, counterbalanced with mobile counter-ions), placed in the electric field. In this paper we present the main electromembrane processes, discuss their mode of operation, their applications and the challenges that they are facing. We then present the latest innovations in the field.

The most common electromembrane process is electrodialysis (ED). A schematic view of the electrodialyzer stack is presented in Fig. 1. A salt solution flows between the alternately placed cation- and anion-exchange membranes. When an electric field is applied in the direction perpendicular to the solution flow, cations migrate through the cation-exchange membranes and are retained by the anion-exchange membranes, whereas the anions migrate through the anion-exchange membranes and are retained by the cation-exchange membranes. Thus, a salt solution stream is being split into desalinated stream (diluate) and concentrated stream (concentrate).

Electrodialysis is widely recognized as a method for brackish water desalination [1, 2]. ED plants can be cheaper than reverse osmosis (RO) plants for a low salinity feeds. There are several reasons why the ED can outperform RO in brackish water desalination. The ionexchange membranes are more resistant to biofouling than RO membranes, less sensitive to chlorine, able to operate at higher feed SDI and are easier to clean up. The basis of the process 
is different: in reverse osmosis and nanofiltration, the energy is proportional to the amount of solvent that needs to be transported across the membrane; in electrodialysis it is quite the opposite - the required energy is proportional to the amount of ions that is need to be transferred. Because brackish water desalination requires the removal of relatively small amount of salt, the ED can be economically competitive. Electrodialysis is also being applied for the treatment of more concentrated solutions. One of the possible applications of electrodialysis is a brine preconcentration method in evaporative salt production [3]. Recently, electrodialysis has been proposed as a new method for production of concentrated sodium chloride solution for the chlor-alkali industry [4-6].

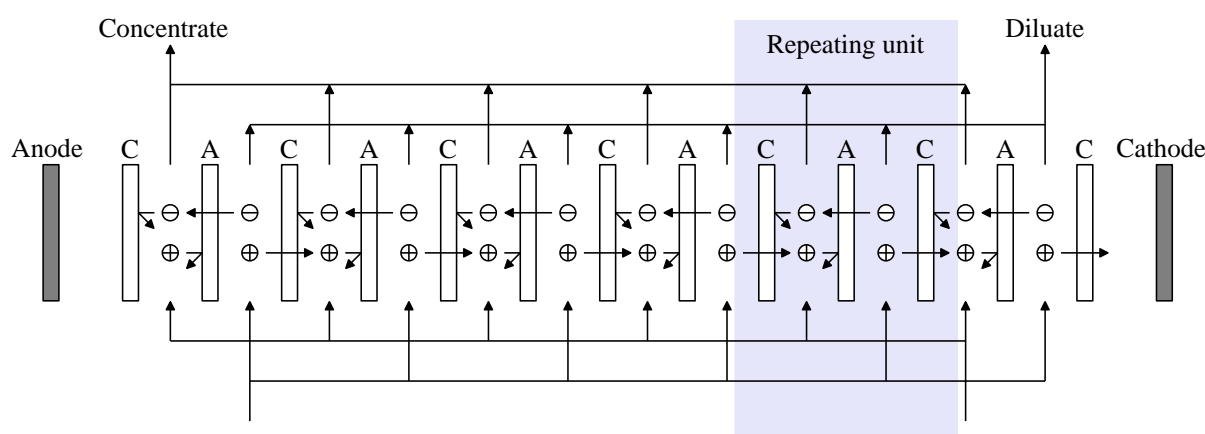

Fig. 1. A scheme of electrodialyzer, electrode reactions not depicted

Electrodialysis faces several challenges. The concentration range of feeds that can be subjected to electrodialysis is limited. On the one hand, during electrodialytic desalination, the decreasing diluate concentration increases the membrane stack resistance. Although small intermembrane distance allow reaching very low diluate concentration [7], the electrodialysis alone cannot economically produce water of very low salinity due to the high investment costs. Electrodialysis becomes inefficient at low salinity due to high electric resistivity. On the other hand, electrodialysis of highly concentrated solutions requires application of high current density, which increases the energy consumption of the process. Working at very high current densities may cause local overheating and chemical damage of the membrane.

Choice of ion-exchange membrane is crucial in design of ED operation. The most important parameters are the electric resistance, permselectivity, chemical stability, and mechanical strength. These parameters often have opposing effect: for example, a high degree of crosslinking improves the mechanical strength, but increases the electrical resistance. More ionic charges in the membrane matrix lead to a low electrical resistance, but in general, cause a high degree of swelling combined with poor mechanical stability [8].

Permselectivity of the ion-exchange membranes describes the ease of counter ions migration through the membrane [8]. Ideally, the membrane should not pass any co-ions; in reality, the amount of co-ions transported through the membrane depends on electrolyte concentration and ion-exchange capacity [8].

The electric resistance of ion exchange membranes is an important factor, especially at high concentration and high current densities. The membrane electric resistance strongly depends on membrane morphology, type of electrolyte and electrolyte concentration and the membrane development focuses on decreasing the resistance by decreasing the membrane thickness.

A relatively recent development in the field of ion-exchange membranes are the univalent permselective membranes and bipolar membranes. Univalent permselective membrane consists of a standard ion-exchange membrane coated with a thin layer of a different charge. 
Such a layer increases the permselectivity towards monovalent ions. The bipolar membrane $(\mathrm{BM})$ is a layered membrane constructed so that one surface is a cation exchange layer, whereas the opposite surface is an anion exchange layer [9]. Between those two layers water splitting occurs, which lowers the $\mathrm{pH}$ of the solution contacting the cation-exchange side of a $\mathrm{BM}$ and increases the $\mathrm{pH}$ of the solution facing the anion-exchange side. Bipolar membranes are used in production of acids and bases from their salts; however, they face low current efficiency and problems with ion leakage.

A big challenge in ED is the concentration polarization and the limiting current density. Because the ion transport number is higher in the membrane than in solution, the ion concentration in layer adjacent to the membrane surface (boundary layer) is different than in the bulk of the solution: lower on the diluate side of the membrane and higher on the concentrate side. As the current density increases, concentration at the diluate side of the membrane drops, eventually reaching zero at the so-called limiting current density (LCD). Working above the LCD can result in electrosplitting of water, which decreases the current efficiency, changes the $\mathrm{pH}$, and may lead to crystallization of sparingly soluble metal hydroxides, i.e. calcium hydroxide, magnesium hydroxide [10]. There are methods of coping with concentration polarization and limiting current density, which have been intensively researched, such as intermembrane spacer design [11-14], adding micro-corrugations on the membrane surface [15], application of conductive spacers [16], introduction of gas bubbles to enhance mixing by creating a two-phase flow [17], or application of pulsed electric field (PEF) [18].

Another important issue in electrodialysis is scaling. The electrodialysis feeds typically contain ions of sparingly soluble salts $\left(\mathrm{Ca}^{2+}, \mathrm{Mg}^{2+}, \mathrm{SO}_{4}{ }^{2-}\right.$, and $\left.\mathrm{HCO}_{3}{ }^{-}\right)$. As the desalination goes on, the salinity of the concentrate increases, increasing the risk of membrane fouling by crystallization of inorganic compounds. Traditionally, anti-scalants, such as etidronic acid, sodium hexametaphosphate, polyamino polyether methylene phosphonic acid are used; but they may be problematic if the concentrate is to be used for salt production or discharged to the environment. Another approach to scaling mitigation is the electrodialysis reversal - an electrodialysis performed with periodic reversal of electrodes' polarity. When the polarity changes, direction of ion migration changes, which tears off the growing deposits and the membrane electric resistance is restored. Novel methods of scaling avoidance are being developed: performed a single-pass electrodialysis in a such manner that residence time of majority of the particles travelling the concentrate compartment is smaller than the crystallization induction time, which prohibits the creation of crystal nuclei inside the ED module [19]; application of pulsed electric field (PEF), which acts as cleaning-in place process $[18,20]$; application of external magnetic field, which disturbs the double ionic layer surrounding the colloidal particles and their zeta potentials by action of Lorentz forces exerted either on moving ions or on charged solid particles - the result is the decreased nucleation rate [21]; application of ultrasonic field, which mechanism of action is still disputed [21].

Since the invention of the electrodialysis there has been a constant development in stack configuration, which resulted in creating new electromembrane processes: electrodialysis metathesis (EDM), electro-electrodialysis (EED), electrodialysis with bipolar membrane (EDBM); however, a lot of progress have been made with hybrid electromembrane processes: electrodeionization (EDI), which combines electrodialysis and ion exchange; membrane capacitive deionization (MCDI) and flow electrode capacitive deionization (FCDI), which combine electrosorption of porous carbon electrodes with ion-exchange membranes; electrochemical desalination, which combines electrochemical reactions with ion-exchange membranes; electrodialysis with ultrafiltration (EDUF) membrane, which combines electrodialysis with pressure-driven methods; and electrodialysis with liquid membranes. 
In an electrodeionization unit, ion exchange resins are placed between the ion selective membranes of the electrodialyzer. This allows in situ resin regeneration due to the creation of hydroxyl and hydrogen ions by the water splitting caused by electric overpotential [9]. Ion exchange resin also increases the conductance of resin-filled compartments. EDI has several advantages over traditional ion exchange: it is a continuous operation, with in situ resin regeneration; it produces no hazardous wastes, as ion-exchange post-regeneration lyes; it removes weakly dissociated species: boric acid, ammonia, silica, carbon dioxide [22]. Similarly to electrodialysis, electrodeionization can be operated in polarity reversal mode in order to mitigate scaling [23] or utilize the bipolar membrane [24, 25] as a source of resinregenerating ions. The drawback of EDI is that it requires high quality feed water, typically having total dissolved salts content (TDS) lower than $25 \mathrm{mg} / \mathrm{dm}^{3}$; thus, sophisticated pretreatment of the feed water is required, which is typically done by reverse osmosis.

Membrane capacitive deionization (MCDI) is a process, which integrates capacitive deionization (CDI) with ion-exchange membranes. The principle of membrane capacitive deionization is presented in Fig. 2. Feed solution flows between porous carbon electrodes with ion-exchange membranes placed on top of them. During sorption step, ions migrate towards the electrodes through the ion-exchange membranes, causing a decrease in solution salinity. When the electrodes are fully loaded, the electrode polarity is reversed or the power is switched off, causing ion desorption. The ion-exchange membrane acts as a barrier, preventing ions desorbed on one electrode from sorption on the second electrode during the desorption step. Recently, a concept of flow electrode capacitive deionization (FCDI) has been introduced: the electrode is a suspension of carbon particles, circulated from cathode to anode compartments of the CDI unit [26]. Although a lot of papers focus on development of new electrode material, the economic viability of MCDI remains questionable.

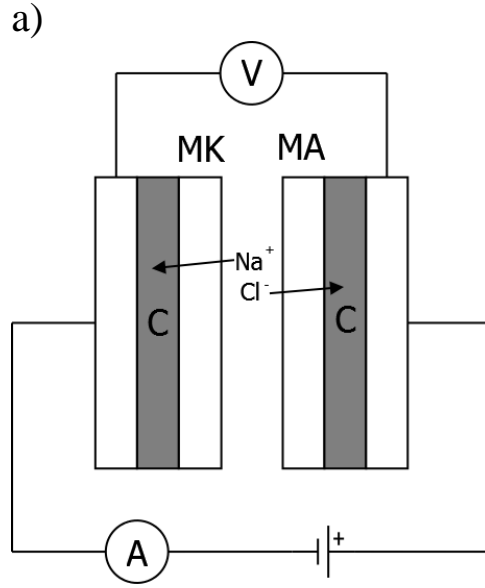

a)

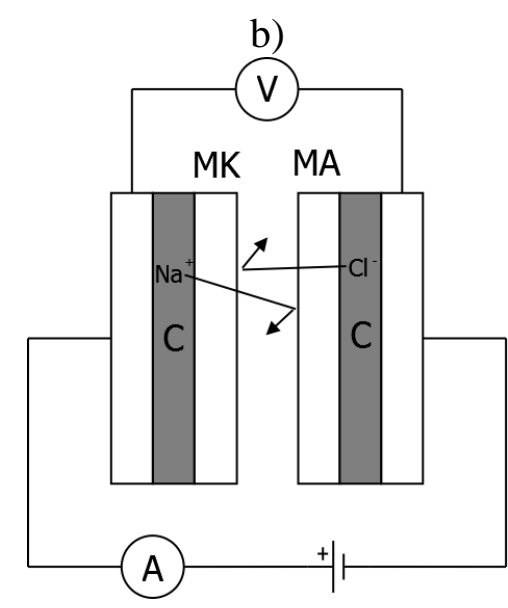

b)

Fig. 2. A scheme of membrane capacitive deionization $M K$-cation-exchange membrane, MA - anion-exchange membrane, $C$ - porous carbon electrode, a) sorption b) desorption

There are some interesting hybrid electromembrane processes being researched. Electrochemical desalination is a recently proposed microfluidic technique, based on Faradaic reactions. In this concept, a positively charged $\mathrm{Ag} / \mathrm{AgCl}$ electrode is used. The chloride anion migrates towards the $\mathrm{Ag} / \mathrm{AgCl}$ electrode and reacts with $\mathrm{Ag}^{+}$created by oxidation of silver to form silver chloride. The sodium cations migrate through cation-exchange membrane towards negatively charged cathode [27]. Electrodialysis with ultrafiltration membrane (EDUF) has been investigated as a method for separating large particles, such as peptides, based on their 
molecular charge and size in an electric field without any pressure. EDUF process has been studied extensively for bioactive peptides separation and concentration from a variety of food proteins, such as $\beta$-lactoglobulin, soy, flaxseed, alfalfa white, whey protein hydrolysates, or for separation of anticancer and antibacterial peptides from snow crab by-product hydrolysates [28]. Electrodialysis with liquid membranes is based on selective transport through ionic liquid placed between the ion-exchange membranes of the electrodialyzer. Since the organic phase can be highly selective toward a specific ion, the membrane-ionic liquid-membrane set acts as a selective membrane - examples include lithium recovery [29].

Finally, there is a large and growing interest in generating electric power with electromembrane processes. This is possible by reverse electrodialysis (RED). A stack design is similar to the conventional ED (see Fig. 1), but instead of applying electrical power to cause the ion flux across the membranes, high salinity solution and a low salinity solution are introduced into the module. Concentration gradient across the membrane causes spontaneous diffusion, migration of ions generates electricity. Plenty of scientists and engineers work on increasing the power density (electric power generated per membrane area) and the method is being heavily researched. However, there are several problems limiting the real-life applications of RED. One of the biggest is where to find both highly concentrated brine and pure water close to each other - this may limit the application of RED geographically. The electric resistance of the membranes is a major obstacle and new, low-resistance membranes dedicated for RED are constantly being developed. Costs of ion-exchange membranes are still too high compared to obtained power. Electric resistance of the solutions can be significant, especially if thick intermembrane spacers are applied; on the other hand, application of thin spacers can increase the hydraulic pressure drop, up to a point where the pumping energy requirement can exceed the generated electric energy, making the device uneconomical. Moreover, the driving force of the process decreases along the membrane as the concentrations of two solutions is slowly converging. Application of very small intermembrane distance can increase the ion flux through the membrane by decreasing the boundary layer thickness and subsequently decreasing the concentration polarization, but it simultaneously means driving force will decrease along the membrane faster, in borderline case making the RED stack impractically short.

In conclusion, electromembrane processes is a growing field of research with a plethora of both existing and still developed applications. The research of electromembrane processes consist in developing new ion-exchange membrane, improving the performance of conventional technologies by enhancing mass transfer and retarding scaling, investigating new stack designs and novel hybrid processes.

\section{LITERATURE}

[1]. Valero F., Arbós R., Desalination of brackish river water using electrodialysis reversal (EDR): control of the THMs formation in the barcelona (NE spain) area, Desalination 253 (2010) 170-174.

[2]. Goodman N. B., Taylor R. J., Xie Z., Gozukara Y., Clements A., A feasibility study of municipal wastewater desalination using electrodialysis reversal to provide recycled water for horticultural irrigation, Desalination 317 (2013) 77-83.

[3]. Turek M., Dydo P., Klimek R., Salt production from coal-mine brine in EDevaporation-crystallization system, Desalination 184 (2005) 439-446.

[4]. Reig M., Casas S., Aladjem C., Valderrama C., Gilbert O., Valero F., Centeno C. M., Larrotcha E., Cortina J. L., Concentration of $\mathrm{NaCl}$ from seawater reverse osmosis brines for the chlor-alkali industry by electrodialysis, Desalination 342 (2014) 107-117. 
[5]. Turek M., Mitko K., Chorążewska M., Dydo P., Use of the desalination brines in the saturation of membrane electrolysis feed, Desalin. Water Treat. 51 (2013) 2749-2754.

[6]. Casas S., Bonet N., Aladjem C., Cortina J. L., Larrotcha E., Cremades L., Modelling sodium chloride concentration from seawater reverse osmosis brine by electrodialysis: Preliminary results, Solvent Extr. Ion Exch. 29 (2011) 488-508.

[7]. Turek M., Mitko K., Bandura-Zalska B., Ciecierska K., Dydo P., Ultra-pure water production by integrated electrodialysis-ion exchange/electrodeionization, Membr. Water Treat. 4 (2013) 237-249.

[8]. Nagarale R., Gohil G., Shahi V. K., Recent developments on ion-exchange membranes and electro-membrane processes, Adv. Colloid Interface Sci. 119 (2006) 97-130.

[9]. Tanaka Y., Ion Exchange Membranes: Fundamentals and Applications, Elsevier, 2015.

[10]. Park J.-S., Song J.-H., Yeon K.-H., Moon S.-H., Removal of hardness ions from tap water using electromembrane processes, Desalination 202 (2007) 1-8.

[11]. Gurreri L., Tamburini A., Cipollina A., Micale G., Ciofalo M., CFD simulation of mass transfer phenomena in spacer filled channels for reverse electrodialysis applications, Chem. Eng. Trans. 32 (2013) 1879-1884.

[12]. Balster J., Pünt I., Stamatialis D. F., Wessling M., Multi-layer spacer geometries with improved mass transport, J. Membr. Sci. 282 (2006) 351-361.

[13]. Turek M., Dydo P., Electrodialysis reversal of calcium sulphate and calcium carbonate supersaturated solution, Desalination 158 (2003) 91-94.

[14]. Gurreri L., Tamburini A., Cipollina A., Micale G., Ciofalo M., CFD prediction of concentration polarization phenomena in spacer-filled channels for reverse electrodialysis, J. Membr. Sci. 468 (2014) 133-148.

[15]. Stroock A. D., Chaotic mixer for microchannels, Science 295 (2002) 647-651.

[16]. Shaposhnik V. A., Grigorchuk O. V., Korzhov E. N., Vasil'eva V. I., Klimov V. Y., The effect of ion-conducting spacers on mass transfer - numerical analysis and concentration field visualization by means of laser interferometry, J. Membr. Sci. 139 (1998) 85-96.

[17]. Wibisono Y., Cornelissen E., Kemperman A., van der Meer W., Nijmeijer K., Twophase flow in membrane processes: A technology with a future, J. Membr. Sci. 453 (2014) 566-602.

[18]. Mikhaylin S., Nikonenko V., Pourcelly G., Bazinet L., Intensification of demineralization process and decrease in scaling by application of pulsed electric field with short pulse/pause conditions, J. Membr. Sci. 468 (2014) 389-399.

[19]. Turek M., Waś J., Mitko K., Scaling prediction in electrodialytic desalination, Desalin. Water Treat. 44 (2012) 255-260.

[20]. Cifuentes-Araya N., Pourcelly G., Bazinet G., How pulse modes affect proton-barriers and anion-exchange membrane mineral fouling during consecutive electrodialysis treatments, J. Colloid Interface Sci. 392 (2013) 396-406.

[21]. Ben Salah Sayadi I., Sistat P., Tlili M. M., Assess of physical antiscale-treatments on conventional electrodialysis pilot unit during brackish water desalination, Chemical Engineering and Processing: Process Intensification 88 (2015) 47-57.

[22]. Wen R., Deng S., Zhang Y., The removal of silicon and boron from ultra-pure water by electrodeionization, Desalination 181 (2005) 153-159.

[23]. Lee H.-J. , Hong M.-K., Moon S.-H., A feasibility study on water softening by electrodeionization with the periodic polarity change, Desalination 284 (2012) 221-227. 
[24]. Grabowski A., Zhang G., Strathmann H., Eigenberger G., Production of high-purity water by continuous electrodeionization with bipolar membranes: influence of concentrate and protection compartment, Sep. Purif. Technol. 60 (2008) 86-95.

[25]. Grabowski A., Zhang G., Strathmann H., Eigenberger G., The production of high purity water by continuous electrodeionization with bipolar membranes: influence of the anionexchange membrane permselectivity, J. Membr. Sci. 281 (2006) 297-306.

[26]. Rommerskirchen A., Gendel Y., Wessling M., Single module flow-electrode capacitive deionization for continuous water desalination, Electrochem. Commun. 60 (2015) 34-37.

[27]. Roelofs S. H., van den Berg A., Odijk M., Microfluidic desalination techniques and their potential applications, Lab Chip 15 (2015) 3428-3438.

[28]. Suwal S., Roblet C., Amiot J., Doyen A., Beaulieu L., Legault J., Bazinet L., Recovery of valuable peptides from marine protein hydrolysate by electrodialysis with ultrafiltration membrane: impact of ionic strength, Food Res. Int. 65 (2014) 407-415.

[29]. Hoshino T., Development of technology for recovering lithium from seawater by electrodialysis using ionic liquid membrane, Fusion Eng. Des. 88 (2013) 2956-2959. 